\title{
Small proximal aortic diameter is associated with higher central pulse pressure and poor outcome in patients with congestive heart failure
}

\author{
Daisuke Kamimura ${ }^{1}$, Kazuaki Uchino ${ }^{1}$, Hideyuki Ogawa ${ }^{1}$, Makoto Shimizu ${ }^{2}$, Tomohiko Shigemasa ${ }^{3}$, \\ Yukiko Morita $^{4}$, Kazuo Kimura ${ }^{5}$ and Satoshi Umemura ${ }^{1}$
}

Background: A small proximal aortic diameter $(A \circ D)$ is thought to be associated with a higher characteristic impedance of the proximal aorta. However, there has been no evidence of a relationship between directly evaluated AoD and directly measured central aortic pressure or the outcome of patients with cardiovascular diseases. Methods and Results: (a) In 135 patients without heart failure (HF), who underwent coronary catheterization, we retrospectively examined the relationship between the AoD and the central aortic pressure or aortic elastance. The AoD adjusted with covariates was inversely correlated with the central pulse pressure (cPP; coefficient $=-0.75 ; P=0.04$; model $R^{2}=0.575$ ) and the effective arterial elastance index (coefficient $=-0.12 ; P=0.001$; model $R^{2}=0.366$ ). (b) In 197 patients who were hospitalized due to HF, we examined the relationship between the AoD (evaluated using echocardiography) and the outcome using a Cox proportional hazard model. Fifty-three patients died from various causes during the follow-up period (2.2 \pm 1.1 years). Multivariable analysis revealed that the AoD remained an independent risk factor for all-cause death (hazard ratio for 1 s.d. increase of the AoD: $0.68,95 \%$ confidence interval: $0.50-0.92, P=0.013$ ) and cardiovascular death (hazard ratio for 1 s.d. increase of the AoD: $0.63,95 \%$ confidence interval: $0.43-0.93, P=0.019$ ). Conclusions: $A$ small AoD was associated with a higher $\mathrm{CPP}$ and aortic stiffening in the patients without HF, as well as with a poor outcome for HF patients. Although it is easy to evaluate the AoD, it may offer important information regarding the pulsatile load and may be useful for risk stratification of HF patients. Hypertension Research (2014) 37, 57-63; doi:10.1038/hr.2013.111; published online 19 September 2013

Keywords: aortic diameter; central blood pressure; heart failure; outcome

\section{INTRODUCTION}

Alterations in the aortic histological or structural characteristics are considered to be associated with the systolic blood pressure or pulse pressure and affect the cardiac afterload. ${ }^{1}$ One of the conventionally accepted mechanisms underlying age-associated changes in the aortic wall is that repetitive pulsations cause fatigue and fracture of the elastin fibers of the central arteries. Consequently, rigid collagenous fibers directly receive the brunt of the blood pressure. ${ }^{2,3}$ This phenomenon causes the entire artery to stiffen and dilate, causing the central aortic pulse pressure to increase, ${ }^{2,3}$

Recently, with respect to the relationship between the aorta and the pulse pressure, there has been a controversy between two theories. O'Rourke et al. ${ }^{2,3}$ have suggested that the stiffening of the aortic wall, as described above, increases the pulse wave velocity (PWV), which causes the reflected wave to return early and ride on top of the forward wave at a later systolic period, amplifying the pulse pressure. However, Mitchell et al..$^{4-7}$ have insisted that an impedance mismatch in the proximal aorta is the main factor driving the increase in pulse pressure in the aged population or in patients with hypertension, and that the reflected wave has only a minor role. They have also suggested that the proxymal aortic diameter (AoD) is a principal determinant of the aortic impedance and that the pulse pressure inversely correlates with the AoD. ${ }^{4-7}$

Other reports have suggested this inverse correlation between the proximal aortic diameter and the peripheral pulse pressure or the indirectly measured central pulse pressure $(\mathrm{cPP}){ }^{8-11}$ However, to date, no studies have evaluated the association between the AoD and the directly measured central pressure, and no studies have evaluated the association between the AoD and the outcome of patients with cardiac diseases. The inverse association between the AoD and the cPP raises the possibility that a small $\mathrm{AoD}$ may increase the aortic pulsatile load; however, longitudinal studies would be indispensable for demonstrating the actual effects of a small AoD on patients with cardiac diseases. If these associations are clarified, the role of the $\mathrm{AoD}$

${ }^{1}$ Department of Medical Science and Cardiorenal Medicine, Yokohama City University, Graduate School of Medicine, Yokohama, Japan; ${ }^{2}$ Department of Cardiology, International Goodwill Hospital, Yokohama, Japan; ${ }^{3}$ Department of Cardiology, International University of Health and Welfare Atami Hospital, Atami, Japan; ${ }^{4}$ Department of Cardiology, National Sagamihara Hospital, Sagamihara, Japan and ${ }^{5}$ Division of Cardiology, Yokohama City University Medical Center, Yokohama, Japan

Correspondence: Dr D Kamimura, Department of Medical Science and Cardiorenal Medicine, Yokohama City University, Graduate School of Medicine, 3-9 Fukura, Kanazawa-ku, Yokohama 236-0004, Japan.

E-mail d.kamimura@cyber.ocn.ne.jp

Received 1 December 2012; revised 3 May 2013; accepted 5 June 2013; published online 19 September 2013 
in the amplification of cardiac afterload will be elucidated, and the present controversy may be resolved. Therefore, the aim of this study was to clarify whether the $\mathrm{AoD}$ is associated with the directly evaluated central blood pressure and whether the AoD is associated with the outcome of patients with heart failure (HF), who are presumably vulnerable to cardiac afterload.

\section{METHODS}

This study consisted of two protocols. The aim of Study 1 was to investigate the relationship between the $\mathrm{AoD}$ and the central aortic pressure or aortic elastance in patients without HF. Study 2 was conducted to assess the effect of the AoD on the outcome of patients with congestive HF.

\section{Study 1}

Patients. From June 2011 to March 2012, consecutive patients who underwent cardiac catheterization to diagnose coronary artery disease were retrospectively assessed for their eligibility to participate in this study. The patients with acute coronary syndrome, significant stenosis of the coronary artery, congestive HF acute myocarditis, congenital heart disease, sick sinus syndrome, complete atrioventricular block, severe valve disease, atrial fibrillation or renal failure (serum creatinine concentration, $>2.5 \mathrm{mg} \mathrm{dl}^{-1}$ ) were excluded from this study.

Hemodynamics and cardiac function. All of the patients were in the fasting state, and medications were not withheld before catheterization. In all the patients, left ventriculography and measurement of the aortic pressure were performed after coronary angiography. The aortic pressure was recorded using a pigtail or multipurpose catheter connected to a fluid-filled transducer. The central aortic pressure (central systolic blood pressure; central diastolic blood pressure; cPP; central mean arterial blood pressure), heart rate, effective arterial elastance index (EaI) and proximal aortic diameter at the end-diastole were determined and analyzed. The EaI was estimated using the end-systolic pressure/stroke volume normalized to the body surface area (BSA). EaI correlates well with the arterial load calculated from aortic impedance data. ${ }^{12}$ The stroke volume varies directly according to the body size; therefore, EaI was adjusted for the BSA. The end-systolic pressure was estimated as the systolic pressure $\times 0.90$. Previous studies demonstrated that this estimation correlates well with the directly measured end-systolic pressure. ${ }^{12,13}$ The cPP was calculated as central systolic blood pressure - central diastolic blood pressure, and the central mean arterial blood pressure was calculated as central diastolic blood pressure $+(\mathrm{cPP} / 3)$. The left ventriculography results for each patient were saved in a local server and evaluated by one researcher. Using the left ventricle (LV) analysis software Cardio Net View-Analytical Review Station, Version 3.2.12.12 (Camtronics Medical Systems, Hartland, WI, USA), we determined the LV volume and stroke volume using the area-length method. The AoD was measured at the level of sinuses of Valsalva on the RAO $30^{\circ}$ view from the left ventriculography.

\section{Study 2}

Patients. This study was a prospective cohort study to determine the factors that affect the prognosis of patients with HF. From August 2003 to November 2006 in Kanagawa prefecture and Shizuoka prefecture in Japan, 251 consecutive patients who were hospitalized because of HF were registered. The clinical diagnosis of HF was based on the Framingham criteria, as previously described. ${ }^{14}$ From the registry described above, the patients with acute myocardial infarction, acute myocarditis, congenital heart disease, significant valvular heart disease, renal failure (serum creatinine concentration, $>2.5 \mathrm{mg} \mathrm{dl}^{-1}$ ), malignancy and chronic inflammatory disease were excluded from this study. Following these exclusion criteria, 197 patients were recruited to participate in this study. All of the registered patients provided their written informed consent before participating in the registry, and the study was approved by the clinical study review board of the Yokohama City University Hospital.

Echocardiography. Echocardiography was performed on the registered patients, within $72 \mathrm{~h}$ of hospital admission. The LV dimensions at end-diastole (LVDd)/end-systole, interventricular septal wall thickness at end-diastole (IVSd), posterior wall thickness at end-diastole (PWd) and AoD were evaluated using the M-mode method. ${ }^{15}$ In this study, the AoD was evaluated at the level of the sinuses of Valsalva. The LV ejection fraction was calculated using the Teichholz method, ${ }^{16}$ and the LV mass index (LVMI) was calculated using the following equation, as recommended by the American Society of Echocardiography: ${ }^{15}$

$$
\begin{aligned}
\text { LVMI }\left(\mathrm{g} \mathrm{m}^{-2}\right)= & (0.8 \times\{1.04 \times[(\mathrm{LVDd}+\mathrm{IVSd} \\
& \left.\left.\left.+\mathrm{PWd})^{3}-(\mathrm{LVDd})^{3}\right]\right\}+0.6\right) / \mathrm{BSA}
\end{aligned}
$$

Laboratory data. Laboratory data obtained immediately before the hospital discharge were used for analysis.

Endpoint. The primary outcome, which was analyzed from the time of registry to the first event, was all-cause death. The secondary outcome was cardiovascular death. Cardiovascular death includes death due to a worsening of HF, sudden death and death due to stroke, aortic diseases, or a worsening of renal function.

\section{Statistical analysis}

All the analyses were performed using the SPSS statistical package version 16.0 (SPSS, Chicago, IL, USA).

Study 1. The correlations between variables were assessed using Pearson's correlation coefficient. A multiple linear regression analysis was performed to assess the independent relationships between the $\mathrm{AoD}$ and the hemodynamic variables. Data are presented as the mean \pm s.d. unless otherwise specified. A value of $P<0.05$ was considered statistically significant.

Study 2. The associations between the analyzed variables and survival were established using a Cox proportional hazards analysis (both univariate and multivariable models). In these analyses, we included the following factors as potential prognosticators: age, gender, body mass index, plasma brain natriuretic peptide concentration, serum sodium concentration, serum creatinine concentration, anemia, AoD (a continuous variable was used for the Cox analysis), LVDd, LV ejection fraction, LVMI and the use of drugs (that is, angiotensin-converting enzyme inhibitors, angiotensin receptor blockers, $\beta$-blockers and aldosterone receptor blockers). The assumptions of the proportional hazard were tested for all variables. Multivariable analysis was performed to assess the independent relationships between the AoD and the covariates. In consideration of the small number of events, we initially set up a model that included a small number of covariates (AoD, age and gender) and then added additional covariates. To illustrate the effect of the AoD on the survival rate, Kaplan-Meier curves for cumulative survival were constructed for two groups of patients with HF classified according to the AoD. Differences in survival rate were tested using the Cox-Mantel log-rank test. The significance of the differences between these two groups was determined using Student's $t$-test. All tests were two-sided. A value of $P<0.05$ was considered statistically significant.

\section{RESULTS}

\section{Study 1}

Baseline characteristics. The baseline characteristics of the patients included in Study 1 are shown in Table 1 . This population was relatively older and included more males than females. As coronary angiography was performed in many of the patients in this study to follow them up after coronary intervention, $\sim 42 \%$ of the patients had a history of ischemic heart disease, and the proportion of patients with hypertension or dyslipidemia was relatively high. Cardiac systolic function was normal in all the participants.

Simple linear regression analysis. The correlations between each hemodynamic variable and the patient characteristics, as well as the correlations between the AoD and the patient characteristics, were evaluated using simple linear regression analysis (Supplementary Table 1). The AoD was significantly and inversely correlated with the central systolic blood pressure, $\mathrm{cPP}$ and EaI. The AoD 
was also significantly correlated with gender, height and weight (Supplementary Table 1).

Multiple linear regression analysis. The AoD adjusted by covariates, including age, gender, height, weight, central mean arterial blood pressure and heart rate, was significantly and inversely correlated with the central aortic systolic pressure, cPP and EaI (Table 2).

Study 2

Baseline characteristics. The baseline characteristics of the patients included in Study 2 are shown in Table 3. The patients were classified into two groups based on a cutoff AoD of $30 \mathrm{~mm}$. The mean age of

Table 1 Baseline characteristics of patients included in Study 1

Number of patients

Age (years)

Male gender (\%)

Body mass index $\left(\mathrm{kg} \mathrm{m}^{-2}\right)$

\section{Medical history}

Hypertension (\%)

Dyslipidemia (\%)

Diabetes mellitus (\%)

Ischemic heart disease (\%)

Laboratory data

Creatinine $\left(\mathrm{mg} \mathrm{dl}^{-1}\right)$

Sodium (meq $\mathrm{I}^{-1}$ )

Hemoglobin $\left(\mathrm{g} \mathrm{dl}^{-1}\right.$ )

LDL cholesterol (mg dl ${ }^{-1}$ )

$\mathrm{HbA1C}(\%)$

\section{Cardiac structure and hemodynamics}

Aortic root diameter at end-diastole $(\mathrm{mm})$

$\mathrm{LV}$ volume at end-diastole ( $\mathrm{mm}$ )

LV volume at end-systole $(\mathrm{mm})$

LV ejection fraction (\%)

Central systolic pressure $(\mathrm{mm} \mathrm{Hg})$

Central diastolic pressure $(\mathrm{mm} \mathrm{Hg})$

Central pulse pressure $(\mathrm{mm} \mathrm{Hg})$

Heart rate (b.p.m.)

Effective arterial elastance index $\left(\mathrm{mm} \mathrm{Hg} \mathrm{ml}^{-1} \mathrm{~mm}^{-2}\right)$

Drugs at catheter

ACEI or ARB (\%)

$\mathrm{BB}(\%)$

CCB (\%)

Statin (\%)

bbreviations: $A C E I$, angiotensin-converting enzyme inhibitor: $A R B$, angiotensin receptor blocker; BB, $\beta$-blocker; CCB, calcium channel blocker; LDL, low-density lipoprotein,

LV, left ventricle.

Values are expressed as mean \pm s.d. or only percentage value. this population was over 70 years. The mean LV ejection fraction was below $40 \%$ and the mean plasma brain natriuretic peptide concentration was greater than $300 \mathrm{pg} \mathrm{ml}^{-1}$; therefore, the majority of these patients suffered from HF with systolic dysfunction. The primary cause for HF was ischemic heart disease in 31\%, dilated cardiomyopathy in 25\% and hypertension in 20\%. A small AoD was more frequently associated with an older age, the female gender and the presence of anemia. However, the other variables did not differ significantly between the two groups. The median follow-up period of this population was 1095 days, and the interquartile range was 593-1095 days.

Univariate analysis. In univariate Cox proportional hazard regression models, the following variables were shown to predict all-cause death: age, low body mass index, the presence of anemia, a low serum sodium concentration, a high plasma brain natriuretic peptide
$69.9 \pm 9.6$

58.5

$23.8 \pm 3.6$

47.4

18.8

42.1

$0.79 \pm 0.21$

$142 \pm 3$

$13.5 \pm 1.4$

$109 \pm 33$

$5.5 \pm 0.7$

$26.4 \pm 2.9$

$70.4 \pm 19.9$

$19.1 \pm 9.8$

$73.6 \pm 8.5$

$139 \pm 22$

$76 \pm 12$

$63 \pm 19$

$75 \pm 13$

$4.28 \pm 1.58$
Table 3 Baseline characteristics of patients included in Study2

\begin{tabular}{lcc}
\hline & $\begin{array}{c}\text { Small AoD } \\
(\mathrm{n}=69)\end{array}$ & $\begin{array}{c}\text { Large AoD } \\
(\mathrm{n}=100)\end{array}$ \\
\hline Age (year) & $72.7 \pm 11.2$ & $67.7 \pm 14.6^{*}$ \\
Male gender (\%) & 37.7 & $73^{*}$ \\
Body mass index $\left(\mathrm{kg} \mathrm{m}^{-2}\right)$ & $21.7 \pm 3.2$ & $23.1 \pm 5.1$
\end{tabular}

Medical history

Hypertension (\%)

Diabetes mellitus (\%)

Dyslipidemia (\%)

Atrial fibrillation (\%)

Ischemic heart disease (\%)

$\begin{array}{ll}41 & 39 \\ 38 & 33 \\ 22 & 20 \\ 33 & 44 \\ 41 & 30\end{array}$

\section{Laboratory data}

Presence of anemia (\%)

59

$38 *$

Serum creatinine $\left(\mathrm{mg} \mathrm{dl}^{-1}\right)$

$1.09 \pm 0.48$

$1.12 \pm 0.45$

Serum sodium (meq $\mathrm{I}^{-1}$ )

$139.1 \pm 3.7$

$139.2 \pm 3.3$

Plasma brain natriuretic peptide $\left(\mathrm{pg} \mathrm{ml}^{-1}\right) \quad 365 \pm 330 \quad 311 \pm 350$

Cardiac structure and hemodynamics

LV dimension at end-diastole $(\mathrm{mm})$

LV ejection fraction ( $\mathrm{mm}$ )

$56.9 \pm 12.7$ AoD (mm)

$42.1 \pm 17.6 \quad 39.4 \pm 16.9$

$26.7 \pm 2.9 \quad 34.5 \pm 3.8^{*}$

Drugs at discharge

ACEI or ARB use (\%)

Beta blocker use (\%)

Aldosterone blocker use (\%)

$\begin{array}{ll}78 & 77 \\ 46 & 40 \\ 43 & 44\end{array}$

Abbreviations: AoD, aortic root diameter; $\mathrm{ACEI}$, angiotensin-converting enzyme inhibitor; $\mathrm{ARB}$, angiotensin receptor blocker; BB, $\beta$-blocker; LV, left ventricle.

Values are expressed as mean \pm s.d. or only percentage value. ${ }^{*} P<0.05$ versus small AoD group.

Table 2 Multiple linear regression analysis in Study 1

\begin{tabular}{|c|c|c|c|c|c|c|c|c|c|c|c|c|}
\hline \multirow[b]{2}{*}{ Variable } & \multicolumn{3}{|c|}{$c S B P$} & \multicolumn{3}{|c|}{$c D B P$} & \multicolumn{3}{|c|}{$c P P$} & \multicolumn{3}{|c|}{ Eal } \\
\hline & $B$ & s.e. & P-values & $B$ & s.e. & P-values & $B$ & s.e. & P-values & $B$ & s.e. & P-values \\
\hline AoD & -0.49 & 0.24 & $0.04 *$ & 0.25 & 0.12 & $0.04 *$ & -0.75 & 0.36 & $0.04 *$ & -0.12 & 0.037 & 0.001 * \\
\hline
\end{tabular}

Abbreviations: AoD, aortic diameter; B, regression coefficient; cSBP, central systolic blood pressure; cDBP, central diastolic blood pressure; cPP, central pulse pressure; Eal, effective arterial elastance index.

${ }^{*} P<0.05$ 
Table 4 Univariate analysis (Cox proportional hazard regression models) in Study 2

\begin{tabular}{|c|c|c|c|c|c|c|}
\hline \multirow[b]{2}{*}{ Variables } & \multicolumn{3}{|c|}{ All-cause death } & \multicolumn{3}{|c|}{ Cardiovascular death } \\
\hline & $H R$ & $95 \% \mathrm{Cl}$ & P-values & $H R$ & $95 \% \mathrm{Cl}$ & P-values \\
\hline Age (year; 1 s.d.) & 1.43 & $1.06-1.93$ & $0.018^{*}$ & 1.40 & $0.98-2.01$ & 0.065 \\
\hline Male gender & 1.17 & $0.67-2.06$ & 0.58 & 0.87 & $0.46-1.69$ & 0.68 \\
\hline Body mass index $\left(\mathrm{kgm}^{-2} ; 1\right.$ s.d. $)$ & 0.55 & $0.37-0.81$ & $0.002^{*}$ & 0.55 & $0.34-0.89$ & $0.013^{*}$ \\
\hline \multicolumn{7}{|l|}{ Laboratory data } \\
\hline Presence of anemia & 2.24 & $1.18-4.23$ & $0.013^{*}$ & 3.05 & $1.33-6.96$ & $0.008^{*}$ \\
\hline Serum creatinine (mg dl ${ }^{-1} ; 1$ s.d.) & 1.29 & $0.99-1.66$ & 0.06 & 1.28 & $0.94-1.76$ & 0.12 \\
\hline Plasma sodium (meq $\mathrm{I}^{-1} ; 1$ s.d.) & 0.70 & $0.54-0.90$ & $0.007^{*}$ & 0.60 & $0.45-0.80$ & $<0.001^{*}$ \\
\hline Plasma brain natriuretic peptide (pg ml-1 $; 1$ s.d.) & 1.52 & $1.23-1.88$ & $<0.001^{*}$ & 1.72 & $1.37-2.17$ & $<0.001 *$ \\
\hline \multicolumn{7}{|l|}{ Echocardiography } \\
\hline AoD (mm; 1 s.d.) & 0.68 & $0.50-0.92$ & $0.013^{*}$ & 0.63 & $0.43-0.93$ & $0.019 *$ \\
\hline LV dimension at end-diastole ( $\mathrm{mm} ; 1$ s.d.) & 0.97 & $0.73-1.28$ & 0.83 & 1.03 & $0.73-1.46$ & 0.87 \\
\hline LV ejection fraction (\%; 1 s.d.) & 0.94 & $0.70-1.26$ & 0.69 & 0.86 & $0.59-1.25$ & 0.43 \\
\hline LV mass index ( $\mathrm{g} \mathrm{mm}^{-2} ; 1$ s.d.) & 0.98 & $0.72-1.33$ & 0.89 & 1.04 & $0.71-1.53$ & 0.83 \\
\hline \multicolumn{7}{|l|}{ Drugs at discharge } \\
\hline ACEI or ARB use & 0.64 & $0.35-1.17$ & 0.15 & 0.58 & $0.28-1.19$ & 0.14 \\
\hline$\beta$-Blocker use & 1.02 & $0.59-1.77$ & 0.95 & 0.86 & $0.44-1.70$ & 0.66 \\
\hline Aldosterone blocker use & 0.63 & $0.36-1.14$ & 0.13 & 0.50 & $0.24-1.07$ & 0.07 \\
\hline
\end{tabular}

Abbreviations: ACEI, angiotensin-converting enzyme inhibitor; ARB, angiotensin receptor blocker; AoD(I), aortic root diameter (index); CI, confidence interval; HR, hazard ratio; LV, left ventricle.

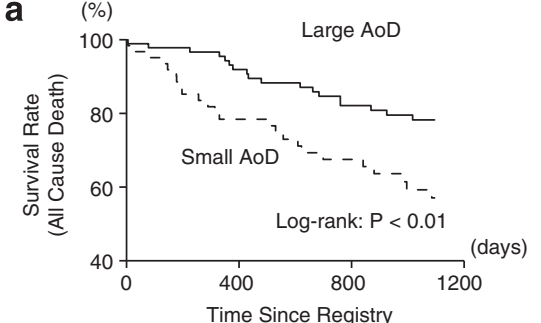

No. at risk

Small AoD $69 \quad 54 \quad 48 \quad 44 \quad 39 \quad 32 \quad 2$

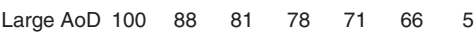

b $(\%)$

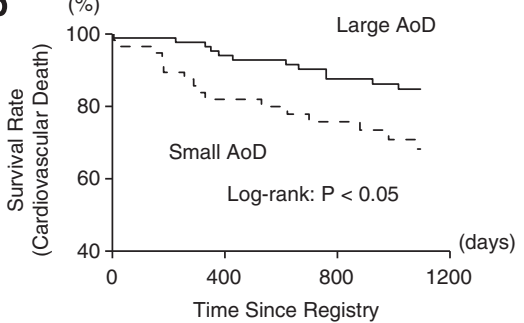

No. at risk

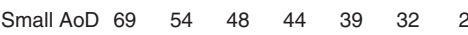

$\begin{array}{lllllll}\text { Large AoD } 100 & 88 & 81 & 78 & 71 & 66 & 5\end{array}$

Figure 1 Kaplan-Meier survival curves of patients in Study 2. (a) Survival curves in which the endpoint was all-cause death. The survival rate was significantly lower in small $A \circ D$ group than in large $A \circ D$ group (log-rank test, $P<0.01$ ). (b) Event-free survival curves in which the endpoints were cardiovascular death. The survival rate was also significantly lower in small AoD group than in large AoD group (log-rank test, $P<0.05$ ). Numbers of patients at risk were described at every 6 months.

concentration and a small AoD (Table 4). These variables, with the exception of age, also predicted cardiovascular death (Table 4). When the studied cohort was divided according to a cutoff AoD of $30 \mathrm{~mm}$, the patients with a small AoD had higher rates of all-cause death and cardiovascular death than did the patients with a large AoD (Figure 1). When all-cause death was considered as an event, the 3 -year event-free survival rates were 57\% (95\% confidence interval: 50-64\%) and 78\% (95\% confidence interval: $74-83 \%)$ in patients with a small AoD and in those with a large $\mathrm{AoD}$, respectively. When cardiovascular death was considered as an event, the 3-year survival rates were $68 \%$ (95\% confidence interval: $62-75 \%)$ and $85 \%$ (95\% confidence interval: $81-89 \%)$ in patients with a small AoD and in those with a large $\mathrm{AoD}$, respectively.

Subgroup analysis. After stratification was performed for the five variables that were associated with all-cause death in the univariate analysis, as well as two added variables (gender and LV ejection fraction), the relationship between the AoD and all-cause death was analyzed and the interaction was examined. The results indicated that no interactions were detected among any of the variables (Supplementary Figure 1).

Multivariable analysis. After adjustment for the covariates in all models, the AoD consistently remained a significant predictor of all-cause death and cardiovascular death in patients with congestive HF (Table 5).

\section{DISCUSSION}

In this study, we examined the relationship between the proximal aortic diameter and the central aortic blood pressure and aortic elastance in patients without HF, and we also examined the relationship between the proximal aortic diameter and the outcome of patients with congestive HF. When adjusted for the covariates, the 
Table 5 Multivariable analysis

a: All-cause death (Cox proportional hazard regression models) in Study 2

\begin{tabular}{|c|c|c|c|c|c|c|c|c|c|c|c|c|c|c|c|}
\hline \multirow[b]{2}{*}{ Variables } & \multirow{2}{*}{$\begin{array}{c}A \\
H R\end{array}$} & \multicolumn{2}{|c|}{ Event $48 / \mathrm{n}=160$} & \multirow{2}{*}{$\frac{B}{H R}$} & \multicolumn{2}{|c|}{ Event $41 / \mathrm{n}=126$} & \multirow{2}{*}{$\begin{array}{c}C \\
H R\end{array}$} & \multicolumn{2}{|c|}{ Event $40 / \mathrm{n}=125$} & \multirow{2}{*}{$\frac{D}{H R}$} & \multicolumn{2}{|c|}{ Event $37 / \mathrm{n}=115$} & \multirow{2}{*}{$\frac{E}{H R}$} & \multicolumn{2}{|c|}{ Event $35 / \mathrm{n}=111$} \\
\hline & & $95 \% \mathrm{Cl}$ & P-values & & $95 \% \mathrm{Cl}$ & P-values & & $95 \% \mathrm{Cl}$ & P-values & & $95 \% \mathrm{Cl}$ & P-values & & $95 \% \mathrm{Cl}$ & P-values \\
\hline Age (1 s.d.) & 1.48 & $1.09-2.01$ & 0.012 & 1.34 & $0.94-1.91$ & 0.103 & 1.39 & $0.92-1.94$ & 0.123 & 1.24 & $0.83-1.84$ & 0.287 & 1.36 & $0.89-2.07$ & 0.15 \\
\hline Male gender & 1.87 & $1.00-3.51$ & 0.049 & 1.47 & $0.73-2.96$ & 0.285 & 1.57 & $0.73-3.12$ & 0.264 & 1.57 & $0.76-3.26$ & 0.223 & 1.46 & $0.69-3.11$ & 0.32 \\
\hline AoD (1 s.d.) & 0.62 & $0.45-0.86$ & 0.004 & 0.58 & $0.40-0.86$ & 0.006 & 0.55 & $0.36-0.81$ & 0.003 & 0.53 & $0.35-0.81$ & 0.003 & 0.53 & $0.34-0.83$ & 0.005 \\
\hline BNP (1 s.d.) & & & & 1.58 & $1.25-2.01$ & $<0.001$ & 1.51 & $1.18-1.95$ & 0.001 & 1.60 & $1.22-2.10$ & $<0.001$ & 1.60 & $1.20-2.12$ & 0.001 \\
\hline $\mathrm{Na}$ (1 s.d.) & & & & & & & 0.71 & $0.52-0.96$ & 0.025 & 0.67 & $0.50-0.92$ & 0.011 & 0.68 & $0.49-0.95$ & 0.021 \\
\hline BMI (1 s.d.) & & & & & & & & & & 0.59 & $0.36-0.96$ & 0.034 & 0.54 & $0.32-0.91$ & 0.021 \\
\hline Anemia & & & & & & & & & & & & & 1.03 & $0.47-2.25$ & 0.95 \\
\hline
\end{tabular}

b: Cardiovascular death (Cox proportional hazard regression models) in Study 2

\begin{tabular}{|c|c|c|c|c|c|c|c|c|c|c|c|c|c|c|c|}
\hline \multirow[b]{2}{*}{ Variables } & \multirow{2}{*}{$\begin{array}{c}A \\
H R\end{array}$} & \multicolumn{2}{|c|}{ Event $31 / \mathrm{n}=155$} & \multirow{2}{*}{$\begin{array}{c}B \\
H R\end{array}$} & \multicolumn{2}{|c|}{ Event $27 / \mathrm{n}=122$} & \multirow{2}{*}{$\begin{array}{c}C \\
H R\end{array}$} & \multicolumn{2}{|c|}{ Event $26 / \mathrm{n}=121$} & \multirow{2}{*}{$\begin{array}{c}D \\
H R\end{array}$} & \multicolumn{2}{|c|}{ Event $24 / \mathrm{n}=112$} & \multirow{2}{*}{$\begin{array}{c}E \\
H R\end{array}$} & \multicolumn{2}{|c|}{ Event $22 / \mathrm{n}=108$} \\
\hline & & $95 \% \mathrm{Cl}$ & P-values & & $95 \% \mathrm{Cl}$ & P-values & & $95 \% \mathrm{Cl}$ & P-values & & $95 \% \mathrm{Cl}$ & P-values & & $95 \% \mathrm{Cl}$ & P-values \\
\hline Age (11 s.d.) & 1.40 & $0.96-2.04$ & 0.084 & 1.24 & $0.80-1.94$ & 0.334 & 1.20 & $0.74-1.95$ & 0.467 & 1.16 & $0.70-1.94$ & 0.562 & 1.39 & $0.79-2.48$ & 0.253 \\
\hline Male gender & 1.37 & $0.64-2.93$ & 0.421 & 1.19 & 0.49-2.91 & 0.694 & 1.12 & $0.43-2.88$ & 0.814 & 1.15 & $0.46-2.88$ & 0.767 & 0.95 & $0.35-2.55$ & 0.919 \\
\hline AoD (1 s.d.) & 0.61 & $0.40-0.92$ & 0.018 & 0.49 & $0.30-0.79$ & 0.004 & 0.46 & $0.28-0.77$ & 0.003 & 0.45 & $0.27-0.77$ & 0.003 & 0.48 & $0.26-0.81$ & 0.007 \\
\hline BNP (1 s.d.) & & & & 1.94 & $1.48-2.55$ & $<0.001$ & 1.83 & $1.37-2.43$ & $<0.001$ & 1.88 & $1.39-2.54$ & $<0.001$ & 1.85 & $1.34-2.56$ & $<0.001$ \\
\hline $\mathrm{Na}$ (1 s.d.) & & & & & & & 0.59 & $0.42-0.83$ & 0.002 & 0.56 & 0.39-0.79 & 0.001 & 0.56 & $0.38-0.82$ & 0.003 \\
\hline BMI (1 s.d.) & & & & & & & & & & 0.57 & $0.31-1.05$ & 0.070 & 0.47 & $0.24-0.93$ & 0.030 \\
\hline Anemia & & & & & & & & & & & & & 1.22 & $0.42-3.58$ & 0.718 \\
\hline
\end{tabular}

Abbreviations: AoD, aortic root diameter; BMI, body mass index; BNP brain natriuretic peptide; $\mathrm{Cl}$, confidence interval; HR, hazard ratio; Na, sodium.

AoD was inversely correlated with the central aortic pulse pressure and EaI. The AoD was also associated with all-cause death and cardiovascular death of patients with congestive HF.

Two conflicting theories explain the amplification of the central aortic load. According to the hypothesis of O'Rourke et al., ${ }^{2,3}$ by the time the aortic elastance increases and the $\mathrm{cPP}$ increases, the central aorta will have been enlarged. In contrast, Mitchel et al. ${ }^{4,5}$ showed that a relatively small aortic diameter contributes to an augmentation of the pulse pressure through an increase in the aortic impedance. The findings of our present study suggest that a small aortic diameter has a significant role in the increase in aortic load and affects the outcome of patients with HF.

There are some reports supporting the hypothesis of Mitchel et al. For example, the data obtained by the Framingham study showed an inverse relationship between the aortic diameter and brachial pulse pressure after adjusting for age. ${ }^{9}$ Agmon et al. ${ }^{10}$ also made similar findings. Farasat et al. ${ }^{8}$ showed an inverse correlation between the directly measured aortic root diameter and the indirectly measured ccPP. However, there have been no reports regarding the association between the directly evaluated proximal aortic diameter and the directly evaluated central aortic pressure. From this perspective, our study provides new information on the relationship between the aortic diameter and the hemodynamics of the central artery.

From the viewpoint of physics, the relationships between the characteristic impedance of the proximal aorta $(\mathrm{Zc})$ and the AoD and between the PWV and the AoD are expressed as follows: 6,17

(a) $\mathrm{Zc}=4 \times \mathrm{PWV} \times \rho / \pi \mathrm{D}^{2}$

(b) $\mathrm{PWV}=[(\mathrm{Et} \times \mathrm{h}) /(2 \rho \times \mathrm{D})]^{1 / 2}$ where $\rho$ is the specific gravity of blood, Et is the Young's modulus, $\mathrm{h}$ is the aortic wall thickness and $\mathrm{D}$ is the aortic diameter).

These equations indicate that the AoD affects both the impedance of the proximal aorta and the PWV but may exert a greater effect on the impedance than on the PWV. Therefore, a small AoD may be more likely to increase the $\mathrm{CPP}$ through an increase in the impedance of the proximal aorta rather than an increase in the PWV. In this study, the AoD was inversely correlated with the EaI, which was well correlated with the measures of arterial load derived from aortic input impedance data. ${ }^{1}$ Therefore, although we were unable to directly evaluate the aortic impedance, a small AoD correlated with a higher cPPs, at least partly through the increase in aortic impedance. However, in Supplementary Table 1 the height is independently correlated with the cPP. Height has been reported to be negatively correlated with the PWV or augmentation index, ${ }^{18}$ and these parameters were not evaluated in this study. Therefore, we cannot conclude that the aortic impedance has a greater influence on the cPP than does the reflected wave in this Study 1.

In Study 2, after adjusting for the variables that were found to be significantly associated with the outcome according to univariate analysis, the AoD still remained an independent predictor of all-cause death and cardiovascular death in patients with congestive HF. There have been no reports on the relationship between the AoD and the outcome of HF patients; therefore, our findings are quite important. Study 1 showed that the AoD was inversely correlated with the variables associated with aortic load. Therefore, the poor outcome of patients with a small aortic diameter in Study 2 might be attributed at least partly to an increase in cardiac afterload.

In Study 2, we examined the interaction between AoD and other variables, including the factors related with all-cause death, LV ejection fraction and gender. As showed in Supplementary Figure 1, 
there were no interactions in any of the analyses; thus, the AoD may exert equal effects in each stratum.

As previously showed, because $\mathrm{Zc}$ and PWV associate with the absolute value of the AoD, simply the absolute value of the AoD may affect the cardiac afterload. When the AoD was standardized to the height (AoDI), AoDI also consistently remained a significant predictor of all-cause death and cardiovascular death in patients with congestive HF (Supplementary Table 2). We used height rather than BSA to adjust the AoD, because height and BSA were reported to be equally correlated with the AoD, ${ }^{19}$ and height was more linear to AoD than was BSA in this study population (data not shown). Therefore, these results indicated that a relatively small AoD may be associated with the prognosis of patients with congestive HF.

The background pathophysiology of our presented data remains speculative. It is well known that the aortic diameter increases with age and that this increase is associated with an increase in the characteristic impedance. $^{2}$ With respect to the pathological background of our study, there are two components. One is the active force associated with the tone of smooth muscle cells of the arterial wall, which is modulated by the sympathetic nervous system or the renin-angiotensin system. The other is the passive force associated with collagen deposition, increasing the cross linking of collagen, or with calcification ${ }^{20}$ of the arterial wall. If arterial stiffness increases due to these factors, a situation may arise in which for a given constant volume in the proximal aorta, the aortic pressure increases while the aortic diameter remains small. As a result, these mechanisms may contribute to a relatively small aortic diameter. Sugawara et al..$^{21}$ reported that an enlargement of the arterial lumen diameter might compensate for the age-related increase in arterial stiffness and limit the deterioration of the buffering capacity of the central artery. This report may support our data and hypothesis.

We cannot deny the possibility that aortic atherosclerosis is associated with a decrease in the aortic diameter, as well as with stroke, a worsening of renal function or coronary events. These indirect associations may explain the association between a small AoD and the poor outcome observed in patients with HF. However, in Study 1 the AoD was negatively correlated with the cPP. The PP is reported to predict coronary events and to be superior to systolic blood pressure or diastolic blood pressure in predicting coronary events. $^{22}$ Therefore, a small AoD was associated with the prognosis of patients with HF possibly through an increase in coronary events. Furthermore, it is also possible that cardiac systolic dysfunction is associated with a low aortic pressure and a small aortic diameter. However, in our Study 1 AoD was not positively correlated but was instead inversely correlated with the central aortic pressure, casting doubt on this theory.

As shown in our study, the AoD was associated with the aortic load and the outcome of cardiac diseases; if the AoD affects the outcome of HF patients, it will be important to develop interventional strategies that modify the AoD and improve the outcome. Mitchel et al. ${ }^{23}$ reported that Omapatrilat (a dual ACE and neutral endopeptidase inhibitor) could decrease the impedance of the proximal aorta, possibly through dilation of the vessel. Otherwise, a cross-link breaker may be effective when an increase in the central aortic impedance is largely responsible for the high central pressure..$^{24,25}$ However, few studies have examined this approach; therefore, such studies should be conducted in the future.

\section{Study limitations}

There are several limitations in this study. First, Study 1 is a retrospective study, and it is presupposed that the patients underwent coronary angiography; therefore, a relatively high percentage of the patients included in this study had a history of coronary heart disease and coronary risk factors. The cardiac systolic function was preserved in all the patients in Study 1. Therefore, the background features of these patients do not necessarily correspond to those in Study 2. Therefore, we should be cautious when drawing conclusions from the results of both studies along a singular line of thought. Second, in Study 1 we were unable to evaluate the PWV or the impedance of the proximal aorta. Third, as the total number of patients and events in Study 2 were small, the number of variables included in the multivariable model needed to be limited. However, in all the multivariable models the AoD remained consistently and significantly associated with the events; therefore, we think it is reliable that AoD will be associated with the prognosis of HF patients. Fourth, as Study 2 was conducted at various hospitals, the machine used for echocardiography and the cardiographer who performed the procedure varied. These limitations may have affected the findings of Study 2. Therefore, it may be necessary to address these limitations in future studies.

\section{CONCLUSIONS}

AoD was inversely correlated with the directly evaluated cPP, which reflects the aortic load. A small AoD was also associated with the outcome of patients with congestive HF. Evaluation of the AoD is simple; however, it may contain important information on the pulsatile load and may be useful for risk stratification of HF patients.

\section{CONFLICT OF INTEREST}

The authors declare no conflict of interest.

1 Redfield MM, Jacobsen SJ, Borlaug BA, Rodeheffer RJ, Kass DA. Age- and genderrelated ventricular-vascular stiffening: a community-based study. Circulation 2005; 112: 2254-2262.

2 O'Rourke MF, Nichols WW. Aortic diameter, aortic stiffness, and wave reflection increase with age and isolated systolic hypertension. Hypertension 2005; 45: 652-658.

3 O'Rourke MF, Hashimoto J. Mechanical factors in arterial aging: a clinical perspective. J Am Coll Cardiol 2007; 50: 1-13.

4 Mitchell GF, Lacourciere Y, Ouellet JP, Izzo JL Jr., Neutel J, Kerwin LJ, Block AJ, Pfeffer MA. Determinants of elevated pulse pressure in middle-aged and older subjects with uncomplicated systolic hypertension: the role of proximal aortic diameter and the aortic pressure-flow relationship. Circulation 2003; 108: 1592-1598.

5 Mitchell GF, Conlin PR, Dunlap ME, Lacourciere Y, Arnold JM, Ogilvie RI, Neutel J, Izzo JL Jr., Pfeffer MA. Aortic diameter, wall stiffness, and wave reflection in systolic hypertension. Hypertension 2008; 51: 105-111.

6 Mitchell GF, Gudnason V, Launer LJ, Aspelund T, Harris TB. Hemodynamics of increased pulse pressure in older women in the community-based Age, Gene/ Environment Susceptibility-Reykjavik Study. Hypertension 2008; 51: 1123-1128.

7 Mitchell GF, Parise H, Benjamin EJ, Larson MG, Keyes MJ, Vita JA, Vasan RS, Levy D. Changes in arterial stiffness and wave reflection with advancing age in healthy men and women: the Framingham Heart Study. Hypertension 2004; 43: 1239-1245.

8 Farasat SM, Morrell CH, Scuteri A, Ting CT, Yin FC, Spurgeon HA, Chen $\mathrm{CH}$ Lakatta EG, Najiar SS. Pulse pressure is inversely related to aortic root diameter implications for the pathogenesis of systolic hypertension. Hypertension 2008; 51 . 196-202.

9 Vasan RS, Larson MG, Levy D. Determinants of echocardiographic aortic root size. The Framingham Heart Study. Circulation 1995; 91: 734-740.

10 Agmon Y, Khandheria BK, Meissner I, Schwartz GL, Sicks JD, Fought AJ, O'Fallon WM Wiebers DO, Tajik AJ. Is aortic dilatation an atherosclerosis-related process? Clinical, laboratory, and transesophageal echocardiographic correlates of thoracic aortic dimensions in the population with implications for thoracic aortic aneurysm formation. J Am Coll Cardiol 2003; 42: 1076-1083.

11 Bella JN, Wachtell K, Boman K, Palmieri V, Papademetriou V, Gerdts E, Aalto T, Olsen MH, Olofsson M, Dahlof B, Roman MJ, Devereux RB. Relation of left ventricular geometry and function to aortic root dilatation in patients with systemic hypertension and left ventricular hypertrophy (the LIFE study). Am J Cardiol 2002; 89: 337-341.

12 Kelly RP, Ting CT, Yang TM, Liu CP, Maughan WL, Chang MS, Kass DA. Effective arterial elastance as index of arterial vascular load in humans. Circulation 1992; 86: 513-521. 
13 Chen CH, Fetics B, Nevo E, Rochitte CE, Chiou KR, Ding PA, Kawaguchi M, Kass DA. Noninvasive single-beat determination of left ventricular end-systolic elastance in humans. J Am Coll Cardiol 2001; 38: 2028-2034.

14 McKee PA, Castelli WP, McNamara PM, Kannel WB. The natural history of congestive heart failure: the Framingham study. N Engl J Med 1971; 285: 1441-1446.

15 Lang RM, Bierig M, Devereux RB, Flachskampf FA, Foster E, Pellikka PA, Picard MH, Roman MJ, Seward J, Shanewise JS, Solomon SD, Spencer KT, Sutton MS, Stewart WJ. Recommendations for chamber quantification: a report from the American Society of Echocardiography's Guidelines and Standards Committee and the Chamber Quantification Writing Group, developed in conjunction with the European Association of Echocardiography, a branch of the European Society of Cardiology. J Am Soc Echocardiogr 2005; 18: 1440-1463.

16 Kronik G, Slany J, Mosslacher H. Comparative value of eight M-mode echocardiographic formulas for determining left ventricular stroke volume. A correlative study with thermodilution and left ventricular single-plane cineangiography. Circulation 1979; 60: 1308-1316.

17 Painter PR. The velocity of the arterial pulse wave: a viscous-fluid shock wave in an elastic tube. Theor Biol Med Model 2008; 5: 15.

18 van Trijp MJ, Bos WJ, Uiterwaal CS, Oren A, Vos LE, Grobbee DE, Bots ML. Determinants of augmentation index in young men: the ARYA study. Eur J Clin Invest 2004; 34: 825-830.

19 Devereux RB, de Simone G, Arnett DK, Best LG, Boerwinkle E, Howard BV, Kitzman D, Lee ET, Mosley TH Jr., Weder A, Roman MJ. Normal limits in relation to age, body size and gender of two-dimensional echocardiographic aortic root dimensions in persons $>$ / $=15$ years of age. Am J Cardiol 2012; 110: 1189-1194.

20 Sekikawa A, Shin C, Curb JD, Barinas-Mitchell E, Masaki K, El-Saed A, Seto TB, Mackey RH, Choo J, Fujiyoshi A, Miura K, Edmundowicz D, Kuller LH, Ueshima H, Sutton-Tyrrell K. Aortic stiffness and calcification in men in a population-based international study. Atherosclerosis 2012; 222: 473-477.

21 Sugawara J, Otsuki T, Maeda S, Tanabe T, Kuno S, Ajisaka R, Matsuda M. Effect of arterial lumen enlargement on carotid arterial compliance in normotensive postmenopausal women. Hypertens Res 2005; 28: 323-329.

22 Franklin SS, Khan SA, Wong ND, Larson MG, Levy D. Is pulse pressure useful in predicting risk for coronary heart Disease? The Framingham heart study. Circulation 1999; 100: 354-360.

23 Mitchell GF, Izzo JL Jr., Lacourciere Y, Ouellet JP, Neutel J, Qian C, Kerwin LJ, Block AJ, Pfeffer MA. Omapatrilat reduces pulse pressure and proximal aortic stiffness in patients with systolic hypertension: results of the conduit hemodynamics of omapatrilat international research study. Circulation 2002; 105: 2955-2961.

24 Vasan RS. Pathogenesis of elevated peripheral pulse pressure: some reflections and thinking forward. Hypertension 2008; 51: 33-36.

25 Vaitkevicius PV, Lane M, Spurgeon H, Ingram DK, Roth GS, Egan JJ, Vasan S, Wagle DR, Ulrich P, Brines M, Wuerth JP, Cerami A, Lakatta EG. A cross-link breaker has sustained effects on arterial and ventricular properties in older rhesus monkeys. Proc Natl Acad Sci USA 2001; 98: 1171-1175.

Supplementary Information accompanies the paper on Hypertension Research website (http://www.nature.com/hr) 